\title{
Polimorfismos Genéticos Associados ao Aparecimento de Hipertensão Arterial Numa População Portuguesa
}

\author{
Genetic Polymorphisms Associated with the Onset of \\ Arterial Hypertension in a Portuguese Population
}

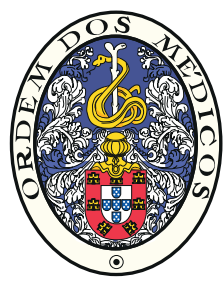

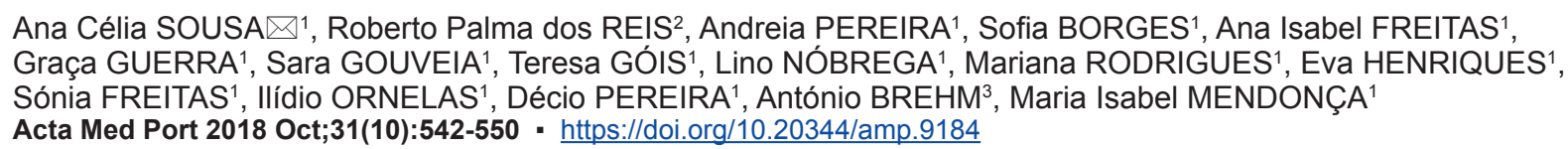

RESUMO

Introdução: A hipertensão arterial é uma doença complexa, multifatorial, controlada por fatores genéticos e ambientais.

Objetivo: Avaliar a susceptibilidade genética no aparecimento de hipertensão arterial e sua associação com os fatores de risco tradicionais na eclosão desta patologia.

Material e Métodos: Estudo caso-controlo com 1712 indivíduos, idade média de 51,0 \pm 7,9 anos (860 hipertensos e 852 controlos). Avaliaram-se os fatores tradicionais, bioquímicos e as variantes genéticas: ACE l/D rs4340, ACE A2350G rs4343, AGT T174M rs4762, AGT M235T rs699 AGTR1 A1166C rs5186, CYP11B2 -344 C/T rs1799998, ADRB1 R389G rs1801253, ADRB2 R16G rs1042713, ADD1 G460W rs4961, SCNN1G G173A rs5718, GNB3 C825T rs5443, ATP2B1 A/G rs2681472, CYP17A1 T/C rs11191548, SLC4A2 $\mathrm{C} / \mathrm{T}$ rs2303934. Calculámos o risco de cada gene para a hipertensão, pelos modelos dominante, recessivo, co-dominante e multiplicativo. Através da regressão logística, avaliámos as variáveis associadas à hipertensão. Elaboraram-se curvas ROC com os fatores tradicionais e posteriormente adicionando as variantes genéticas associadas com hipertensão. Analisámos os dados através do SPSS for Windows 19.0 e MedCalc v. 13.3.3.0.

Resultados: As variantes genéticas ADD1 G460W, GNB3 C825T, ACE I/D e ACE A2350G associaram-se à hipertensão. A curva ROC com os factores de risco tradicionais e estas variantes mostrou um incremento na capacidade preditiva de hipertensão $(p=0,018)$.

Discussão: Segundo os resultados do nosso estudo as variantes genéticas que após análise univariada se associaram à hipertensão arterial foram a ACE I/D rs4340, ACE A2350G rs4343, ADD1 G460W rs4961, GNB3 C825T rs5443. As duas primeiras variantes relacionam-se com a hipertensão arterial por interferirem no sistema renina-angiotensina-aldosterona, que tem um importante papel na regulação da pressão arterial. Salienta-se o facto dos genes que codificam os componentes do sistema renina-angiotensinaaldosterona serem candidatos naturais ao desenvolvimento e progressão da hipertensão arterial. Também na nossa população os polimorfismos da alfa-aducina (ADD1 G460W rs4961), associaram-se à hipertensão arterial. Nesta população portuguesa, conhecida por ter elevado consumo de sal, faz sentido que estes polimorfismos, sejam relevantes na gestão do sal e da água e consequentemente, no aparecimento de hipertensão arterial. A variante genética GNB3 C825T rs5443 que interfere na sinalização intracelular também constituiu uma forte candidata à hipertensão arterial. Com a elaboração da curva ROC e cálculo das AUC inicialmente só com os fatores de risco tradicionais e posteriormente adicionando as variantes ADD1 G460W, GNB3 C825T, ACE I/D e ACE A2350G aos fatores de risco tradicionais, verificámos ter havido um incremento no risco preditivo de hipertensão arterial, relativamente ao existente só com os fatores de risco tradicionais, com significado estatístico $(p=0,018)$. Isto sugere que a hipertensão arterial é uma doença multifatorial, que resulta da interação de fatores ambientais, genéticos e estilos de vida que interagem entre si e levam ao aparecimento desta importante patologia.

Conclusão: No nosso estudo os polimorfismos associados à hipertensão, estão ligados ao eixo renina-angiotensina-aldosterona ( $A C E$ I/D, ACE A2350G), bem como à gestão de sal e água (ADD1 G460W, GNB3 C825T). Através de uma análise multivariada, concluiuse que estas duas últimas variantes genéticas conjuntamente com quatro dos fatores tradicionais (tabagismo, hábitos alcoólicos, obesidade e diabetes) se associam de forma significativa e independente à hipertensão arterial essencial. Num modelo preditivo de hipertensão arterial, a introdução das variantes genéticas aumenta ligeiramente o valor preditivo do modelo.

Palavras-chave: Factores de Risco; Hipertensão; Polimorfismo Genético; Portugal

\section{ABSTRACT}

Introduction: Arterial hypertension is a complex, multifactorial disease, controlled by genetic and environmental factors.

Objective: Evaluate the genetic susceptibility for developing arterial hypertension and its association with the traditional risk factors in the outbreak of this pathology.

Material and Methods: Case-control study with 1712 individuals, mean age of $51.0 \pm 7.9$ years (860 hypertensive patients and 852 controls). Biochemical and traditional risk factors, and genetic variants were evaluated: ACE I/D rs4340, ACE A2350G rs4343, AGT T174M rs4762, AGT M235T rs699 AGTR1 A1166C rs5186, CYP11B2 -344 C/T rs1799998, ADRB1 R389G rs1801253, ADRB2 R16G rs1042713, ADD1 G460W rs4961, SCNN1G G173A rs5718, GNB3 C825T rs5443, ATP2B1 A/G rs2681472, CYP17A1 T/C rs11191548, SLC4A2 C/T rs2303934. The risk of each gene for hypertension was estimated by the dominant, recessive, co-dominant and multiplicative models. By logistic regression, variables associated with hypertension were evaluated. ROC curves were first performed with traditional risk factors and then adding the genetic variants associated with hypertension. Data were analyzed by SPSS for Windows 19.0 and MedCalc v. 13.3.3.0.

Results: The genetic variants ADD1 G460W, GNB3 C825T, ACE I/D, ACE A2350G were associated with hypertension. ROC curve

1. Unidade de Investigação. Hospital Doutor Nélio Mendonça. Funchal. Portugal.

2. Faculdade de Ciências Médicas. Universidade Nova de Lisboa. Lisboa. Portugal.

3. Laboratório de Genética Humana. Universidade da Madeira. Funchal. Portugal.

$\triangle$ Autor correspondente: Ana Célia Sousa. anacelia.bett@gmail.com

Recebido: 15 de maio de 2017 - Aceite: 10 de julho de 2018 | Copyright @ Ordem dos Médicos 2018 
with traditional risk factors and these variants showed an increase in the predictive capacity of hypertension $(p=0.018)$.

Discussion: According to the results of our study, the genetic variants found to be associated with hypertension were: $A C E$ I/D rs4340, ACE A2350G rs4343, ADD1 G460W rs4961 and GNB3 C825T rs5443. The first two variants are associated with hypertension by interfering with the renin-angiotensin-aldosterone system, which plays an important role in regulating blood pressure. It should be noted that genes encoding the components of renin-angiotensin-aldosterone system are natural candidates for the development and progression of hypertension. In our population alpha-aducin polymorphism (ADD1 G460W rs4961) was also associated with hypertension. In a Portuguese population, known to have high salt intake, it makes sense that this polymorphism which is relevant in salt and water management may consequently be relevant in the onset of hypertension. The genetic variant GNB3 C825T rs5443 that affects intracellular signalling was also found to be a strong risk candidate for hypertension. Initially, with the elaboration of the ROC curve and calculation of the AUC using only with traditional risk factors and later by adding the variants ADD1 G460W, GNB3 C825T, ACE I/D and ACE A2350G to the traditional risk factors, we verified that genetic polymorphisms increased the predictive risk of hypertension, when compared to the risk given only by traditional risk factors, with statistical significance $(p=0.018)$. This suggests that hypertension is a multifactorial disease that results from the interaction of environmental, genetic and lifestyle factors that interact with each other and lead to the advent of this important pathology.

Conclusion: In our study, the hypertension-associated polymorphisms are linked to the renin-angiotensin-aldosterone axis $(A C E$ I/D, ACE A2350G), as well as to salt and water management (ADD1 G460W, GNB3 C825T). Through a multivariate analysis, it was concluded that these two last genetic variants together with four of the traditional risk factors (smoking, alcohol consumption, obesity and diabetes) are associated in a significant and independent way with essential hypertension. In a predictive model of hypertension, the introduction of genetic variants slightly increases the predictive value of the model.

Keywords: Hypertension; Polymorphism, Genetic; Portugal; Risk Factors

\section{INTRODUÇÃO}

A hipertensão arterial (HTA) é um importante problema de saúde pública que afeta mais de um bilião de pessoas em todo o mundo, sendo um fator de risco para enfarte do miocárdio, acidente vascular cerebral e doença renal crónica. ${ }^{1} \mathrm{Na}$ população adulta portuguesa, a sua prevalência global é de $42 \%$, sendo de $44,4 \%$ nos homens e de $40,2 \%$ nas mulheres. ${ }^{2}$

O principal obstáculo para a compreensão dos mecanismos patogénicos da hipertensão arterial humana, decorre da complexidade e heterogeneidade da doença. De fato, embora no nível clínico o quadro pareça bastante uniforme, uma variedade de fatores poligénicos e ambientais poderão estar envolvidos na sua génese. ${ }^{3} \mathrm{~A}$ maioria dos fatores ambientais que influenciam a pressão arterial foi já identificada, ${ }^{4,5}$ mas os fatores genéticos são pouco conhecidos. Vários estudos confirmam a natureza multigénica da pressão arterial em geral ${ }^{6,7}$ e da hipertensão arterial essencial em particular. ${ }^{8}$ Estima-se que os fatores genéticos sejam responsáveis por cerca de $30 \%$ da variação tensional em várias populações. ${ }^{9}$

Com o aparecimento dos estudos de associação genómica em larga escala (GWAS), muitos se têm dedicado à HTA essencial. Em 2009, dois estudos de associação genómica (GWAS), cada um com mais de 25000 indivíduos de ascendência europeia, identificaram 13 loci associados à pressão arterial sistólica (PAS), diastólica (PAD) e hipertensão arterial. ${ }^{10,11} \mathrm{Um}$ outro estudo feito numa população afro-americana, evidenciou associações entre vários genes e a pressão arterial sistólica. ${ }^{12}$ Mais recentemente, em 2011 são relatados pelo Consórcio Internacional de Pressão Arterial (ICBP), 29 variantes genéticas independentes, identificadas pelo GWAS que influenciam a pressão arterial (PA) em pessoas de ascendência europeia. ${ }^{13}$

A identificação de genes candidatos que medeiam a suscetibilidade para a HTA, continua a ser objeto de estudo, principalmente utilizando estudos de associação em seres humanos, mas também utilizando estudos fisiológicos e farmacológicos de intervenção em modelos animais e em estirpes geneticamente modificadas. Sendo assim novas abordagens genéticas, avançam no sentido da compreensão e estudo dos genes que codificam os componentes das vias biológicas, fisiológicas e funções celulares da HTA. ${ }^{10}$

Foram incluídos no presente estudo, polimorfismos genéticos de loci biologicamente plausíveis para HTA, nomeadamente: ACE I/D rs4340, ACE A2350G rs4343, AGT T174M rs4762, AGT M235T rs699 AGTR1 A1166C rs5186, CYP11B2 -344 C/T rs1799998, ADRB1 R389G rs1801253, ADRB2 R16G rs1042713, ADD1 G460W rs4961, SCNN1G G-173A rs5718, GNB3 C825T rs5443, ATP2B1 A/G rs2681472, CYP17A1 T/C rs11191548, SLC4A2 C/T rs2303934.

O objetivo do presente estudo foi avaliar os polimorfismos genéticos que se associam ao aparecimento de hipertensão arterial essencial numa população portuguesa.

\section{MATERIAL E MÉTODOS \\ População do estudo}

O estudo foi realizado no Arquipélago da Madeira (Portugal), cuja população é de cerca de 300000 habitantes. Todos os participantes incluídos no estudo são de raça caucasiana, naturais do Arquipélago da Madeira, sendo os seus progenitores na $1^{\mathrm{a}}$ e $2^{\mathrm{a}}$ gerações igualmente caucasianos e nascidos neste arquipélago.

Este estudo foi aprovado pela Comissão de Ética do Hospital Central do Funchal e conduzido de acordo com a Declaração de Helsínquia. O consentimento informado de cada participante foi obtido por escrito, com permissão explícita para as análises bioquímicas e de ADN bem como para a colheita de dados clínicos relevantes.

Fizemos um estudo caso-controlo com 1712 indivíduos com média de idade de $51,0 \pm 7,9$ anos, sendo $51 \%$ do género masculino, selecionados das consultas de Medicina Geral e Familiar e da Consulta de Medicina Interna do Hospital Central do Funchal. Constituímos dois grupos, um com 860 indivíduos hipertensos (idade média 51,4 \pm 8; 53,3\% do género masculino) e outro com 852 controlos (idade 
média $50,7 \pm 7,7 ; 48,7 \%$ do género masculino). Os controlos foram emparelhados com os casos em termos de sexo e idade.

\section{Definição de hipertensão arterial}

A hipertensão arterial foi considerada quando os pacientes, à entrada do estudo, já estavam diagnosticados e/ ou tinham medicação anti-hipertensiva há pelo menos três meses ou quando diagnosticados com PAS e PAD $\geq 140 / 90$ $\mathrm{mmHg}$ medido em pelo menos três ocasiões. ${ }^{14}$

Os controlos normotensos nunca tinham sido tratados com medicação anti-hipertensiva e apresentaram PAS e $\mathrm{PAD}<140 / 90 \mathrm{mmHg}$.

A pressão arterial foi medida, após 10 minutos de repouso, no braço direito, posição de sentado, usando um esfigmomanómetro Welch Allyn padrão (fases I a V). Registou-se a média de três leituras obtidas com dois minutos de intervalo. ${ }^{14}$

Excluíram-se do estudo os indivíduos com hipertensão secundária, insuficiência de órgãos múltiplos, distúrbios mentais, doença inflamatória crónica, grávidas ou doentes medicados para outras patologias com fármacos que pudessem afetar a pressão arterial.

\section{Recolha de dados}

Todos os participantes preencheram um questionário padronizado com referência à idade, sexo, estilo de vida, tabagismo, consumo de álcool e terapêutica medicamentosa.

A altura foi medida em centímetros e obtido o peso em quilogramas. Calculámos o índice de massa corporal (IMC), utilizando a fórmula (IMC = peso, kg/altura, $\mathrm{m}^{2}$ ). A obesidade foi definida como índice de massa corporal $(\mathrm{IMC})>30$ $\mathrm{kg} / \mathrm{m} .^{15}$

Definiu-se diabetes quando o nível de glicose em jejum $\geq 126 \mathrm{mg} / \mathrm{dL}$ ou o nível de glicose $\geq 200 \mathrm{mg} / \mathrm{dL}$ e/ou existia uma história de terapêutica para diabetes. ${ }^{16}$

Considerou-se o sedentarismo quando os indivíduos não praticavam pelo menos 150 minutos por semana de atividade física moderadamente vigorosa. ${ }^{17}$

Considerou-se o tabagismo e o alcoolismo quando os indivíduos tinham estes hábitos à data de entrada do estudo.

\section{Análise bioquímica}

As análises bioquímicas foram realizadas no laboratório central do Hospital, de acordo com as técnicas usuais.

As amostras de sangue foram extraídas após 14 - 16 horas de jejum e o plasma foi preparado para a quantificação dos perfis biológicos. As concentrações plasmáticas de glicose, colesterol total, triglicéridos, colesterol das lipoproteínas de alta densidade (HDL-C) e colesterol das lipoproteínas de baixa densidade (LDL-C) foram medidas utilizando métodos padronizados no laboratório central do Hospital.

\section{Análise genética}

Extraímos o ADN genómico das células leucocitárias do sangue periférico usando o método standard de salting-out. Efetuámos a análise genotípica usando sondas oligonucleotídicas marcadas com fluorescência específica para cada um dos alelos num ensaio que combina a técnica convencionada de PCR e a técnica TaqMan (Applied Biosystems). Os primers e as sondas foram as pré-estabelecidas pelo fornecedor (TaqMan SNP Genotyping Assays, Applied Biosystems) para os 14 polimorfismos genéticos ACE I/D rs4340, ACE A2350G rs4343, AGT T174M rs4762, AGT M235T rs699, AGTR1 A1166C rs5186, CYP11B2 -344 C/T rs1799998, ADRB1 R389G rs1801253, ADRB2 R16G rs1042713, ADD1 G460W rs4961, SCNN1G G-173A rs5718, GNB3 C825T rs5443, ATP2B1 A/G rs2681472, CYP17A1 T/C rs11191548, SLC4A2 C/T rs2303934. Os oligonucleótidos foram sintetizados e os marcadores fluorogénicos FAM e VIC acoplados às extremidades 5' das sondas de modo a alcançar a discriminação alélica. A reacção de polimerização em dois passos consistiu em 40 ciclos de desnaturação a $92^{\circ} \mathrm{C}$ durante 15 segundos e de primer annealing e extensão a $60^{\circ} \mathrm{C}$ durante um minuto e foi efectuada num aparelho 7300 real-time PCR System (Applied Biosystems). Os genótipos foram determinados recorrendo ao 7300 System SDS Software (Applied Biosystems) sem qualquer conhecimento prévio dos dados clínicos correspondentes.

\section{Análise estatística}

As variáveis contínuas foram expressas pela média \pm desvio padrão nas variáveis de distribuição normal, ou, se tal não aconteceu pela mediana (mínimo - máximo). Para comparar estas variáveis, entre casos e controlos foi utilizado o teste $t$ de Student ou Mann-Whitney. As variáveis categóricas foram apresentadas através da frequência e percentagem e comparadas pelo teste qui-quadrado.

$\mathrm{Na}$ análise multivariada, para verificar quais as variáveis associadas independentemente com a HTA, fizemos uma regressão logística em que entraram as seguintes variáveis: obesidade, diabetes, hábitos tabágicos e alcoólicos, sedentarismo e as variantes genéticas que, na análise univariada, se associaram de forma significativa com a HTA: ADD1 G460W rs4961, GNB3 C825T rs5443, ACE I/D rs4340 e ACE A2350G rs4343. Para a seleção da ordem de entrada das variáveis no modelo foi usando o método Forward Wald.

Para avaliar o risco preditivo de HTA, elaborámos curvas ROC (receiver operating characteristic) e cálculo das respectivas AUC (area under the curve) inicialmente só com os fatores de risco tradicionais (FRT), e posteriormente adicionando as variantes ADD1 G460W, GNB3 C825T, ACE I/D e ACE A2350G aos FRT. As duas curvas foram comparadas pelo Delong test. ${ }^{18}$ Fizemos a calibração dos modelos pelo teste de Hosmer Lemeshow.

Analisámos os dados estatísticos recorrendo ao SPSS (Statistical Package for the Social Sciences Software version 19.0) (IBM, Armonk, NY, USA) e ao software MedCalc 
versão 13.3.3.0.

O limiar de significância foi de $p<0,05$. Todas as análises foram bilaterais.

\section{RESULTADOS}

\section{Caraterísticas gerais da população estudada}

A população estudada foi composta por 860 casos com HTA, com idade média de 51,4 \pm 8 (53.3\% sexo masculino), e 852 controlos sem HTA), com idade média de $50,7 \pm 7,7$ (48,7\% sexo masculino).

As caraterísticas basais da nossa amostra são apresentadas na Tabela 1. O sexo e a idade foram semelhantes nos dois grupos pelo ajustamento das populações de acordo com a metodologia do estudo. Comparando o grupo dos casos com o dos controlos (Tabela 1), houve uma diferença significativa em relação às seguintes variáveis que apresentaram valores médios mais elevados no grupo dos hipertensos em relação aos controlos: sedentarismo $(p=0,018)$, hábitos alcoólicos $(p=0,019)$, diabetes $(p<$ $0,0001)$, obesidade $(p<0,0001)$, pressão arterial sistólica $(p<0,0001)$, pressão arterial diastólica $(p<0,0001)$ e frequência cardíaca $(p=0,001)$. Em relação ao tabagismo, verificámos uma maior percentagem no grupo dos controlos em relação aos hipertensos e com significância estatística $(p=0,001)$.

Em relação às variáveis bioquímicas (Tabela 2), houve uma diferença significativa entre ambos os grupos relativamente à hemoglobina, leucócitos, triglicerídeos, glicose em jejum e proteína $\mathrm{C}$ reativa de alta sensibilidade ( $P C R$ as) que apresentaram valores médios mais elevados no grupo dos hipertensos em relação aos controlos ( $p<0,0001)$. Os níveis de $\mathrm{HDL}$ foram mais baixos nos hipertensos do que nos controlos $(p<0,0001)$. Em relação ao colesterol total, LDL e plaquetas não houve diferença significativa entre os dois grupos.

Variantes genéticas que se associaram com o aparecimento de HTA

Avaliámos as 14 variantes genéticas, mas apenas qua- tro mostraram associação com o aparecimento de HTA (Tabela 3). Nomeadamente, os polimorfismos da: alfa-aducina ADD1 G460W rs4961 nos modelos recessivo $(p=0,003)$ e co-dominante $(p=0,004)$; os da subunidade $\beta 3$ da proteína G (GNB3 C825T rs5443) nos modelos dominante $(p=0,004)$ e multiplicativo $(p=0,044)$; os da enzima de conversão da angiotensina ( $A C E$ I/D rs4340) nos modelos recessivo $(p=0,032)$ e multiplicativo $(p=0,025)$ e os da ACE A2350G rs4343 nos modelos recessivo $(p=0,036)$, multiplicativo ( $p=0,023)$ e co-dominante $(p=0,028)$.

Posteriormente fizemos uma análise de regressão logística (Tabela 4) com as quatro variáveis genéticas que, na análise univariada, se associaram à HTA e com os FRT como a obesidade, diabetes, hábitos tabágicos e alcoólicos e sedentarismo. As variáveis que permaneceram na equação e que se associam de forma significativa e independente com o aparecimento de HTA foram: obesidade $(p<0,0001)$, diabetes $(p<0,0001)$, hábitos alcoólicos $(p=$ $0,013)$ e o genótipo WW do gene $A D D 1(p=0,013)$ e o CT do GNB3 $(p=0,010)$.

Os hábitos tabágicos surgem na equação como variável protetora em relação ao aparecimento de $\operatorname{HTA}(p=0,001)$.

Elaborámos uma curva $R O C$ e calculámos a AUC primeiramente com os FRT e, posteriormente, adicionámos os polimorfismos genéticos que, na análise univariada, se associaram com a HTA (Fig. 1). Verificamos que a adição dos quatro polimorfismos genéticos aos FRT acrescentou um valor preditivo $(A U C)$ de 0,668 para 0,681 , com significado estatístico de acordo com o Delong test $(p=0,018)$.

\section{DISCUSSÃO}

O nosso estudo demonstrou que, após análise univariada, as variantes genéticas $A D D 1 \mathrm{G} 460 \mathrm{~W}$ rs4961, GNB3 C825T rs5443, ACE I/D rs4340, ACE A2350G rs4343 relacionam-se com a HTA, sendo que as variantes $A D D 1$ G460W rs4961, GNB3 C825T rs5443 associam-se de forma significativa e independente com o aparecimento deste patologia na nossa população.

Tabela 1 - Caraterísticas demográficas e clínicas dos participantes do estudo

\begin{tabular}{|c|c|c|c|c|}
\hline Variáveis & $\begin{array}{c}\text { Total } \\
(\mathrm{n}=1712)\end{array}$ & $\begin{array}{l}\text { Hipertensos } \\
(\mathrm{n}=860)\end{array}$ & $\begin{array}{l}\text { Controlos } \\
(n=852)\end{array}$ & Valor $p$ \\
\hline Idade (anos) & $51 \pm 7,9$ & $51,4 \pm 8$ & $50,7 \pm 7,7$ & 0,069 \\
\hline Sexo masculino, n (\%) & $873(51)$ & $458(53,3)$ & $415(48,7)$ & 0,060 \\
\hline Sedentarismo, n (\%) & $915(53,4)$ & $484(56,3)$ & $431(50,6)$ & 0,018 \\
\hline Hábitos alcoólicos, n (\%) & $634(37)$ & $342(39,8)$ & $292(34,3)$ & 0,019 \\
\hline Tabagismo, n (\%) & $392(22,9)$ & $169(19,7)$ & $223(26,2)$ & 0,001 \\
\hline Diabetes, n (\%) & $203(11,9)$ & $163(19)$ & $40(4,7)$ & $<0,0001$ \\
\hline Obesidade, n (\%) & $457(26,7)$ & $330(38,4)$ & $127(14,9)$ & $<0,0001$ \\
\hline $\operatorname{IMC}\left(\mathrm{kg} / \mathrm{m}^{2}\right)$ & $27,7 \pm 4,8$ & $29,1 \pm 5,2$ & $26,2 \pm 4$ & $<0,0001$ \\
\hline PAS $(\mathrm{mmHg})$ & $134,1 \pm 20,5$ & $147,5 \pm 19$ & $120,7 \pm 11$ & $<0,0001$ \\
\hline PAD $(\mathrm{mmHg})$ & $84,2 \pm 12,2$ & $91 \pm 11,9$ & $77,2 \pm 7,9$ & $<0,0001$ \\
\hline FC (bat./min.) & $72 \pm 11,7$ & $72,9 \pm 12,2$ & $71 \pm 11,1$ & 0,001 \\
\hline
\end{tabular}

IMC: Índice de massa corporal; PAS: Pressão arterial sistólica; PAD: Pressão arterial diastólica; FC: Frequência cardíaca; bat./min.: Batimentos/minuto; valores contínuos expressos pela média \pm desvio padrão; valores significativos para $p<0,05$. 
Tabela 2 - Caraterísticas bioquímicas dos participantes do estudo

\begin{tabular}{|c|c|c|c|c|}
\hline Variáveis & $\begin{array}{c}\text { Total } \\
(\mathrm{n}=1712)\end{array}$ & $\begin{array}{l}\text { Hipertensos } \\
(n=860)\end{array}$ & $\begin{array}{l}\text { Controlos } \\
(n=852)\end{array}$ & Valor $p$ \\
\hline Hemoglobina (g/dL) & $14,2(9,6-18,2)$ & $14,4(9,6-18,2)$ & $14,2(10,1-17,6)$ & $<0,0001$ \\
\hline Plaquetas $\left(10^{3} / \mu \mathrm{L}\right)$ & $229(23-664)$ & $233(23-664)$ & $228(65-544)$ & 0,099 \\
\hline Leucócitos $\left(10^{3} / \mu \mathrm{L}\right)$ & $6,4(2,1-16,6)$ & $6,7(2,9-14,8)$ & $6,3(2,1-16,6)$ & $<0,0001$ \\
\hline Colesterol (mg/dL) & $206(100-370)$ & $206,5(100-346)$ & $206(107-370)$ & 0,776 \\
\hline HDL-C (mg/dL) & $48(17,2-111,7)$ & $47(17,2-104)$ & $49,0(20,8-111,7)$ & $<0,0001$ \\
\hline LDL-C (mg/dL) & $129(37,7-269)$ & $128,4(37,7-269)$ & $130(42-260)$ & 0,082 \\
\hline Triglicéridos (mg/dL) & $111(21-1098)$ & $122,5(29-1098)$ & $100(21-688)$ & $<0,0001$ \\
\hline PCR-as (mg/dL) & $0,22(0,01-19,62)$ & $0,23(0,02-19,62)$ & $0,2(0,01-18,51)$ & $<0,0001$ \\
\hline Glicose (mg/dL) & $96(66-364)$ & $99(70-360)$ & $94(66-364)$ & $<0,0001$ \\
\hline
\end{tabular}

HDL-C: colesterol das lipoproteínas de alta densidade; LDL-C: Colesterol das lipoproteínas de baixa densidade; PCR-as: Proteína C reactiva-alta sensibilidade; valores apresentados pela mediana (mínimo - máximo); valores significativos para $p<0,05$.

Tabela 3 - Variantes genéticas que se associaram com o aparecimento de hipertensão arterial

\begin{tabular}{cccccccc}
\hline \multirow{2}{*}{ Genes } & & Casos & Controlos & \multicolumn{4}{c}{ Modelos Genéticos } \\
\cline { 7 - 8 } & & $(\mathbf{n}=\mathbf{8 6 0})$ & $(\mathbf{n = 8 5 2 )}$ & Dominante & Recessivo & Multiplicativo & Co-dominante \\
\hline \multirow{2}{*}{ ACE } & II & $110(12,8)$ & $128(15)$ & 1,205 & 1,233 & 1,173 & 1,327 \\
rs4340 & ID & $368(42,8)$ & $389(45,7)$ & $(0,916-1,586)$ & $(1,018-1,495)$ & $(1,020-1,348)$ & $(0,989-1,780)$ \\
& DD & $382(44,4)$ & $335(39,3)$ & $p=0,182$ & $p=0,032$ & $p=0,025$ & $p=0,059$ \\
ACE & AA & $143(16,6)$ & $167(19,6)$ & 1,222 & 1,244 & 1,170 & 1,369 \\
rs4343 & AG & $424(49,3)$ & $435(51,1)$ & $(0,955-1,564)$ & $(1,015-1,526)$ & $(1,022-1,340)$ & $(1,035-1,811)$ \\
& GG & $293(34,1)$ & $250(29,3)$ & $p=0,110$ & $p=0,036$ & $p=0,023$ & $p=0,028$ \\
ADD1 & GG & $600(69,8)$ & $617(72,4)$ & 1,138 & 2,763 & 1,202 & 2.805 \\
rs4961 & GT & $230(26,7)$ & $224(26,3)$ & $(0,923-1,402)$ & $(1,376-5,551)$ & $(0,999-1,446)$ & $(1,393-5,647)$ \\
& TT & $30(3,5)$ & $11(1,3)$ & $p=0,226$ & $p=0,003$ & $p=0,051$ & $p=0,004$ \\
GNB3 & CC & $286(33,3)$ & $341(40)$ & 1,339 & 0,998 & 1,152 & 1,201 \\
rs5443 & CT & $440(51,2)$ & $378(44,4)$ & $(1,100-1,631)$ & $(0,769-1,296)$ & $(1,004-1,321)$ & $(0,902-1,600)$ \\
& TT & $134(15,6)$ & $133(15,6)$ & $p=0,004$ & $p=0,987$ & $p=0,044$ & $p=0,210$ \\
\hline
\end{tabular}

ACE: Enzima conversora de angiotensina; $A D D 1$ : Alfa-aducina; GNB3: Subunidade $\beta 3$ da proteína $\mathrm{G}$.

Duas dessas variantes, ACE I/D e ACE A2350G, interferem no sistema renina-angiotensina-aldosterona (SRAA) que tem um importante papel na regulação da pressão arterial, por regular a hemodinâmica renal e a volemia. ${ }^{19}$

Assim, os genes que codificam os componentes do SRAA são candidatos naturais ao desenvolvimento e progressão da HTA. ${ }^{20}$

O gene ACE I/D humano localiza-se no cromossoma $17 q 23$ e é um polimorfismo de inserção/deleção (I/D) de 287 pb no intrão 16 e tem sido utilizado extensivamente como marcador genético. ${ }^{21} \mathrm{~A}$ enzima conversora da angiotensina (ECA) tem um papel chave na geração da angiotensina II e degradação da bradicinina e através destas, afeta a morfologia e a reatividade da parede vascular, assim como a função renal. Os níveis plasmáticos individuais da ECA na população normal são reproduzíveis e estáveis, havendo no entanto uma ampla variabilidade inter-individual. O polimorfismo do gene ACE I/D modula fortemente o nível de ECA plasmática, ${ }^{22,23}$ sendo que cerca de metade dessa variabilidade pode ser explicada por este polimorfismo. ${ }^{24}$ Alguns estudos descreveram o alelo $D$ como fator de risco para hipertensão arterial em várias populações..$^{25,26}$
Numa amostra constituída por 3095 participantes do Framingham Heart Study, foi verificada uma associação do genótipo DD com aumento da pressão arterial diastólica em homens, mas não em mulheres. ${ }^{27}$ Num outro estudo populacional, o locus da ACE foi associado à pressão arterial diastólica e à pressão arterial média em adolescentes, com idade média de 15 anos. ${ }^{28}$ Numa meta-análise Ji LD, Zhang $L N$ et a ${ }^{29}$ reportou que o genótipo DD do polimorfismo $A C E$ I/D associou-se com a hipertensão com um risco de 1,61 e através de modelos genéticos dominantes, recessivos e aditivos, também foram encontradas associações positivas. ${ }^{29}$ No entanto, outros estudos não comprovam esta associação. ${ }^{30-32}$

Assim, o presente estudo reforça, numa população portuguesa, o conceito de que o genótipo DD da ACE se associa, de forma significativa, com a HTA. O odds ratio encontrado foi, no entanto, relativamente baixo, na ordem de 1,2, o que sugere uma influência isolada relativamente modesta e pode explicar que noutros estudos, nomeadamente com amostras de dimensão inferior, esta diferença não atinja a significância.

Um outro polimorfismo do gene ACE, uma substituição 
Tabela 4 - Análise de regressão logística* para predição da doença hipertensiva

\begin{tabular}{lcc}
\hline Variáveis & Odds ratio (IC 95\%) & Valor $\boldsymbol{p}$ \\
\hline Fumador & $0,662(0,518-0,845)$ & 0,001 \\
Álcool & $1,306(1,058-1,613)$ & 0,013 \\
Obesidade & $3,138(2,469-3,989)$ & $<0,0001$ \\
Diabetes & $3,510(2,404-5,126)$ & $<0,0001$ \\
ADD1 & ----- & 0,044 \\
GW & $1,048(0,834-1,317)$ & 0,687 \\
WW & $2,527(1,219-5,239)$ & 0,013 \\
GN 33 & ----- & 0,030 \\
CT & $1,339(1,073-1,671)$ & 0,010 \\
TT & $1,283(0,947-1,739)$ & 0,108 \\
\hline
\end{tabular}

* Método Forward Wald (SPSS v. 19.0) em que saíram da equação as seguintes variáveis: sedentarismo; genes ACE8 e ACE ID. IC: Intervalo de confiança; estatisticamente significativo para $p<0,05$.

A/G na base 2350 do exão 17 (A2350G) foi estudado por Zhu et a ${ }^{\beta 3}$ numa amostra populacional da Nigéria com 1343 indivíduos. Neste estudo, este polimorfismo associou-se a uma maior concentração de ACE plasmática e à pressão sanguínea. ${ }^{33}$ Saeed et $a^{\beta 4}$ também concluiu que o genótipo ACE GG estava associado com o aparecimento de HTA (OR = 1,80; IC a 95\% 1,06 - 3,07; $p=0,02)$.
Mas outros estudos apresentaram resultados inconclusivos. ${ }^{35} \mathrm{Na}$ nossa população, confirmamos a importância do polimorfismo da ACE A2350G na HTA. Numa meta-análise com 1699 casos e 1274 controlos, Niu et a/ ${ }^{36}$ concluiu que o alelo ACE 2350A, conferia uma redução significativa do risco de hipertensão nos muçulmanos do Golfo Arábico e do Paquistão e um risco elevado de HTA na população Chinesa Han. ${ }^{36}$

O gene da alfa-aducina ADD1 G460W rs496, localizado no cromossoma $4 p 16.3$, tem sido associado com o aumento da suscetibilidade de desenvolver hipertensão, ${ }^{37-41}$ mas existem resultados contraditórios. ${ }^{42-44}$ A aducina é uma proteína heterodimérica do citoesqueleto, presente em muitos tecidos, com subunidades $\alpha, \beta$ e $y$ envolvidas no contato célula a célula, transporte de iões da membrana celular e transdução de sinal. É uma das proteínas que regulam $\mathrm{Na}+$ - $\mathrm{K}+$ ATPase. ${ }^{45,46} \mathrm{~A}$ variante genética mutada ADD1 460W, leva à alteração da actina e têm demonstrado influenciar a expressão superficial e a velocidade máxima da $\mathrm{Na}+$ - $\mathrm{K}+$ ATPase e subsequentemente uma mais rápida reabsorção de $\mathrm{Na}+$ tubular renal e a um aumento da pressão sanguínea. ${ }^{47}$ No presente trabalho, os polimorfismos da alfa-aducina, quer no modelo recessivo, quer no co-dominante, apresentaram um risco importante de HTA, com um OR de 2,8. Numa população conhecida por ter elevado consumo

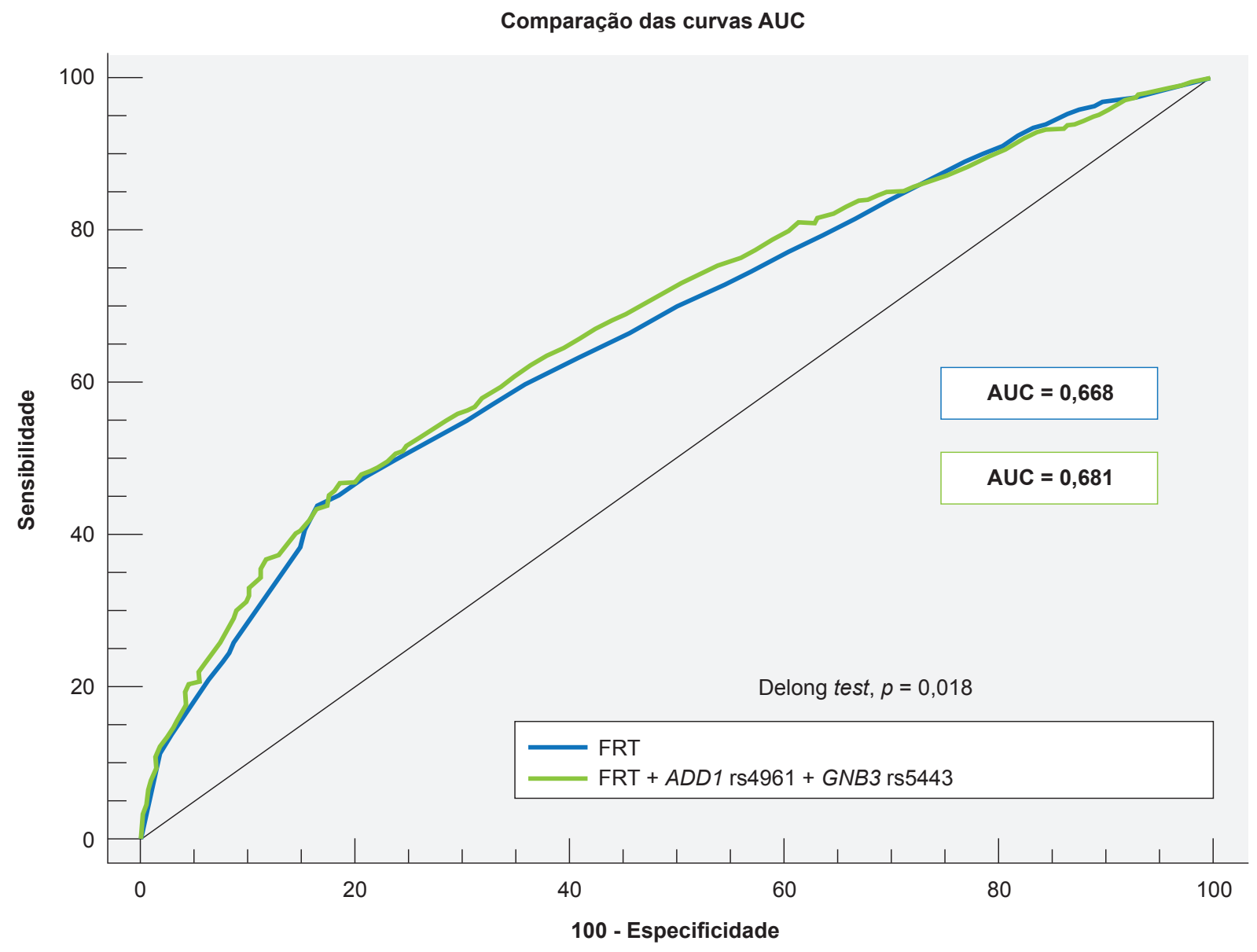

Figura 1 - Curvas ROC realizadas para os FRT e para os FRT com as variantes genéticas 
de sal, faz sentido que estes polimorfismos sejam relevantes na gestão do sal e da água, e, consequentemente, no aparecimento de HTA.

Vários investigadores descreveram uma associação do polimorfismo da subunidade $\beta 3$ da Proteína G C825T rs5443 com a HTA, ${ }^{48-51}$ mas outros estudos não confirmam este resultado. ${ }^{52,53} \mathrm{O}$ gene $\beta 3$ da proteína $\mathrm{G}$, está localizado no cromossoma $12 \mathrm{p} 13 .{ }^{54}$ O polimorfismo GNB3 C825T resulta dum splicing alternativo do exão 9 que elimina 41 aminoácidos na proteína, associando-se assim à expressão de uma nova variante truncada (G3-s) que se correlaciona com a ativação da proteína G. ${ }^{55}$ Esta variante truncada é uma proteína funcional mas confere uma ativação aumentada das proteínas $\mathrm{G}$, facilitando a sinalização intracelular. ${ }^{55}$ Além do que, uma atividade aumentada da proteína $\mathrm{G}$, aumenta a troca dos iões de $\mathrm{Na}+/ \mathrm{H}+$ e associa-se a maiores níveis plasmáticos de $\mathrm{Na}+$ e menores de potássio. Isto evidencia o papel dessas proteínas na patogénese da $\mathrm{HTA} .{ }^{56} \mathrm{O}$ trabalho que apresentamos revela uma influência modesta destes polimorfismos no aparecimento da HTA, estatisticamente significativo nos modelos dominante e multiplicativo, com riscos na ordem de 1,2 a 1,3.

Adicionalmente, os nossos resultados mostram que, após análise de regressão logística, as variáveis que permaneceram na equação, demonstrando estarem associadas ao aparecimento de HTA de forma significativa e independente, foram a obesidade $\mathrm{OR}=3,138$, hábitos alcoóli$\cos \mathrm{OR}=1,306$, diabetes $\mathrm{OR}=3,510$ e os genótipos WW da $A D D 1$ rs4961 OR $=2,527$ e o CT do GNB3 rs5443 com $\mathrm{OR}=1,339$.

Curiosamente, os hábitos tabágicos aparecem como um fator protetor, ou seja, nos controlos havia mais indivíduos com hábitos tabágicos do que no nos hipertensos $(26.2 \%$ vs $19.7 \%$ ). Este resultado aparentemente paradoxal pode explicar-se pelo facto dos indivíduos hipertensos já estarem a ser seguidos em consultas médicas e em abordagem geral do risco cardiovascular muitos deixaram de fumar.

Após elaboração da curva ROC e cálculo das AUC inicialmente só com os FRT e posteriormente adicionando as variantes $A D D 1 \mathrm{G} 460 \mathrm{~W}, \mathrm{GNB} 3 \mathrm{C} 825 \mathrm{~T}, A C E \mathrm{I} / \mathrm{D}$ e $A C E$ A2350G aos FRT, verificámos ter havido um incremento no risco preditivo de HTA, relativamente ao existente só com os FRT, com significado estatístico $(p=0,018)$.

Estes resultados sugerem que a HTA é uma doença multifatorial, causada por fatores ambientais, genéticos e estilos de vida que interagem entre si e levam ao aparecimento desta patologia.

\section{Pontos fortes e limitações do estudo}

O presente estudo é o primeiro caso-controlo feito na população da Ilha da Madeira, uma população portuguesa, geneticamente homogénea ${ }^{57-59}$ e relativamente isolada, em que se investiga a associação de variantes genéticas anteriormente relacionadas por estudos de ligação génica ou por GWAS, com a suscetibilidade para a HTA essencial.

Esta população, com as características citadas, representa uma mais-valia para o mapeamento de distúrbios raros e além disso, segundo vários investigadores, o estudo de populações culturalmente e geneticamente isoladas que apresentam um estilo de vida, hábitos alimentares e ambiente natural idênticos, pode reduzir a variação ambiental. ${ }^{60}$

No entanto o nosso estudo avalia um número limitado de variantes genéticas associadas à HTA. A inclusão de mais variantes genéticas poderão reforçar ou acrescentar novos resultados.

\section{CONCLUSÃO}

Os polimorfismos associados ao aparecimento de HTA no presente estudo estão ligados ao eixo renina-angiotensina-aldosterona, nomeadamente ACE I/D e ACE A2350G, bem como os polimorfismos ligados à gestão do sal e água, como os polimorfismos da alfa aducina ( $A D D 1 \mathrm{G} 460 \mathrm{~W}) \mathrm{e}$ da subunidade $\beta 3$ da proteína $\mathrm{G}$ (GNB3 C825T).

A análise multivariada detetou uma associação significativa entre as variantes genéticas $A D D 1$, GNB3 e os fatores de risco tradicionais de HTA na capacidade para predizer o risco de HTA. O presente estudo destaca a complexidade da HTA e demonstra a importância da associação entre fatores comportamentais e fatores genéticos no aparecimento da HTA.

\section{PROTECÇÃO DE PESSOAS E ANIMAIS}

Os autores declaram que os procedimentos seguidos estavam de acordo com os regulamentos estabelecidos pelos responsáveis da Comissão de Investigação Clínica e Ética e de acordo com a Declaração de Helsínquia da Associação Médica Mundial.

\section{CONFIDENCIALIDADE DOS DADOS}

Os autores declaram ter seguido os protocolos do seu centro de trabalho acerca da publicação de dados.

\section{CONFLITOS DE INTERESSE}

Os autores declaram não terem qualquer conflito de interesse relativamente ao presente artigo.

\section{FONTES DE FINANCIAMENTO}

Os autores declaram não ter recebido subsídios ou bolsas para a elaboração do artigo.

treatment and control of hypertension and salt intake in Portugal: changes over a decade. The PHYSA study. J Hypertens. 2014;32:121121.

3. Camussi A, Bianchi G. Genetics of essential hypertension. From the unimodal-bimodal controversy to molecular technology. Hypertension. 1988;12:620-8.

\section{REFERÊNCIAS}

1. Lim SS, Vos T, Flaxman AD, Danaei G, Shibuya K, Adair-Rohani H et al. A comparative risk assessment of burden of disease and injury attributable to 67 risk factors and risk factor clusters in 21 regions, 1990 2010: a systematic analysis for the Global Burden of Disease Study 2010. Lancet. 2012;380:2224-60.

2. Polonia J, Martins L, Pinto F, Nazaré J. Prevalence, awareness, 
4. MacGregor GA. Sodium is more important than calcium in essential hypertension. Hypertension. 1985;7:628-40.

5. Harrap SB. Genetic analysis of blood pressure and sodium balance in spontaneously hypertensive rats. Hypertension. 1986;8:572-82.

6. Levy D, DeStefano AL, Larson MG, O'Donnell CJ, Lifton RP, Gavras H, et al. Evidence for a gene influencing blood pressure on chromosome 17: genome scan linkage results for longitudinal blood pressure phenotypes in subjects from the Framingham Heart Study. Hypertension. 2000;36:477-83.

7. Allayee H, de Bruin TW, Dominguez KM, Cheng LS-C, Ipp E, Cantor $\mathrm{RM}$, et al. Genome scan for blood pressure in Dutch dyslipidemic families reveals linkage to a locus on chromosome $4 \mathrm{p}$. Hypertension. 2001;38:773-8.

8. Sharma $\mathrm{P}$, Fatibene J, Ferraro $\mathrm{F}$, Jia $\mathrm{H}$, Monteith $\mathrm{S}$, Brown $\mathrm{C}$, et al. A genome-wide search for susceptibility loci to human essential hypertension. Hypertension. 2000;35:1291-6.

9. Beevers G, Lip GY, O'Brien E. ABC of hypertension: The pathophysiology of hypertension. BMJ. 2001;322:912-6.

10. Newton-Cheh C, Johnson T, Gateva V, Tobin MD, Bochud M, Coin L, et al. Eight blood pressure loci identified by genome-wide association study of 34,433 people of European ancestry. Nat Genet. 2009;41:66676.

11. Levy D, Ehret GB, Rice K, Verwoert GC, Launer LJ, Dehghan A, et al. Genome-wide association study of blood pressure and hypertension. Nat Genet. 2009:41:677-87.

12. Adeyemo A, Gerry N, Chen G, Herbert A, Doumatey A, Huang H, et al. A genome-wide association study of hypertension and blood pressure in African Americans. PLoS Genet. 2009;5:e1000564.

13. Ehret GB, Munroe PB, Rice KM, Bochud M, Johnson AD, Chasman $\mathrm{DI}$, et al. Genetic variants in novel pathways influence blood pressure and cardiovascular disease risk. The International Consortium for Blood Pressure Genome-Wide Association Studies. Nature. 2011;478:103-9.

14. 2003 European Society of Hypertension-European Society of Cardiology guidelines for the management of arterial hypertension. J Hypertens. 2003;21:1011-53.

15. National Institute of Health, National Heart, Lung, and Blood Institute North American Association for the Study of Obesity. The Practical Guide: Identification, Evaluation, and Treatment of Overweight and Obesity in Adults. NHLBI Obesity Education Initiative, NIH Publication Number: 00-4084, 2000.

16. Report of the Expert Committee on the Diagnosis and Classification of Diabetes Mellitus. Diabetes Care. 2003;26:3160-7.

17. 2016 European Guidelines on Cardiovascular disease prevention in clinical practice. Eur Heart J. 2016;37:2315-81.

18. DeLong ER, DeLong DM, Clarke-Pearson DL. Comparing the areas under two or more correlated receiver operating characteristic curves: a nonparametric approach. Biometrics. 1988;44:837-45.

19. Weir MR, Dzau VJ. The renin-angiotensin-aldosterone system: a specific target for hypertension management. Am J Hypertens. 1999;12:205S-13.

20. Ruster C, Wolf G. Renin-Angiotensin-Aldosterone System and Progression of Renal Disease. J Am Soc Nephrol. 2006;17:2985-91.

21. Rigat $B$, Hubert $C$, Corvol P, Soubrier F. PCR detection of the insertion/ deletion polymorphism in the human angiotensin converting enzyme gene (DCP1) (dipeptidyl carboxypeptidase 1). Nucleic Acids Res. 1992;20:1433.

22. Cambien F, Costerousse O, Tiret L, Poirier O, Lecerf L, Gonzales MF, et al. Plasma level and gene polymorphism of angiotensin-converting enzyme in relation to myocardial infarction. Circulation. 1994;90:669-76.

23. Rigat B, Hubert C, Alhenc-Gelas F, Cambien F, Corvol P, Soubrier F. An insertion/deletion polymorphism in the angiotensin l-converting enzyme gene accounting for half the variance of serum enzyme levels. J Clin Invest. 1990;86:1343-6.

24. Danser AH, Schalekamp MA, Bax WA, van den Brink AM, Saxena $\mathrm{PR}$, Riegger $\mathrm{GA}$, et al. Angiotensin-converting enzyme in the human heart. Effect of the deletion/ insertion polymorphism. Circulation. 1995;92:1387- 8

25. Higashimori K, Zhao Y, Higaki J, Kamitani A, Katsuya T, Nakura J, et al. Association analysis of a polymorphism of the angiotensin converting enzyme gene with essential hypertension in the Japanese population. Biochem Biophys Res Commun. 1993;191:399-404.

26. Duru K, Farrow S, Wang JM, Lockette W, Kurtz T. Frequency of a deletion polymorphism in the gene for angiotensin converting enzyme is increased in African-Americans with hypertension. Am J Hypertens. 1994;7:759-62.

27. O'Donnell CJ, Lindpaintner K, Larson MG, Rao VS, Ordovas JM,
Schaefer EJ, et al. Evidence for association and genetic linkage of the angiotensin-converting enzyme locus with hypertension and blood pressure in men but not women in the Framingham Heart Study [see comments]. Circulation. 1998; 97:1766-72.

28. Fornage M, Amos Cl, Kardia S, Sing CF, Turner ST, Boerwinkle E. Variation in the region of the angiotensin-converting enzyme gene influences interindividual differences in blood pressure levels in young white males [comment]. Circulation. 1998;97:1773-9.

29. Lin-dan J, Li-na Z, Peng S, Ping W, Yue-miao Z, Wen-hua X, et al. Association of angiotensinogen gene M235T and angiotensin-converting enzyme gene I/D polymorphisms with essential hypertension in Han Chinese population: a meta-analysis. J Hypertens. 2010;28:419-28.

30. Jeunemaitre X, Lifton RP, Hunt SC, Williams RR, Lalouel JM. Absence of linkage between the angiotensin converting enzyme locus and human essential hypertension. Nat Genet. 1992;1:72-5.

31. Gu XX, Spaepen M, Guo C, Fagard R, Amery A, Lijnen P, et. al. Lack of association between the I/D polymorphism of the angiotensin-converting enzyme gene and essential hypertension in a Belgian population. J Hum Hypertens. 1994;8:683-5.

32. Chiang FT, Lai ZP, Chern TH, Tseng CD, Hsu KL, Lo HM, et al. Lack of association of the angiotensin converting enzyme polymorphism with essential hypertension in a Chinese population. Am J Hypertens. 1997; 10:197-201.

33. Zhu X, Bouzekri N, Southam L, Cooper RS, Adeyemo A, McKenzie CA, et al. Linkage and association analysis of angiotensin I - converting enzyrne (ACE)-gene polymorphisms with ACE concentration and blood pressure. Am J Hum Genet. 2001;68:1139-48.

34. Saeed MM, Saboohi K, Osman Ali S, Bokhari AM, Frossard PM. Association of the angiotensin-converting enzyme (ACE) gene G2350A dimorphism with essential hypertension. J Hum Hypertens. 2003;17:71923.

35. Niu W, Qi Y, Hou S, Zhai X, Zhou W, Qiu C. Haplotype -based association of the renin-angiotensin-aldosterone system genes polymorphisms with essential hypertension among Han Chinese: the Fangshan study. J Hypertens. 2009;27:1384-91.

36. Niu W, Qi Y, Gao P, Zhu D. Association between angiotensin converting enzyme G2350A polymorphism and hypertension risk: a meta-analysis. J Renin Angiotensin Aldosterone Syst. 2011;12:8-14.

37. Cusi D, Barlassina C, Azzani T, Casari G, Citterio L, Devoto M, et al. Polymorphisms of alfa-adducin and salt sensitivity in patients with essential hypertension. Lancet.1997;349:1353-7.

38. Tamaki S, Iwai N, Tsujita Y, Nakamura Y, Kinoshita M. Polymorphism of alpha-adducin in Japanese patients with essential hypertension. Hypertens Res. 1998;21:29-32.

39. Casari G, Barlassina C, Cusi D, Zagato L, Muirhead R, Righetti M, et al. Association of the alfa adducin locus with Essential Hypertension. Hypertension. 1995;25:320-6.

40. Barlassina C, Norton GR, Samani NJ, Woodwiss AJ, Candy GC, Radevski I, et al. Alpha-adducin polymorphism in hypertensives of South African ancestry. Am J Hypertens. 2000;13:719-23.

41. Ju Z, Zhang H, Sun K, Song $Y$, Lu H, Hui R, et al. Alpha-adducin gene polymorphism is associated with essential hypertension in Chinese: a case-control and family-based study. J Hypertens. 2003;21:1861-8.

42. Ishikawa K, Katsuya T, Sato N, Nakata Y, Takami S, Takiuchi S, et al. No association between alpha-adducin 460 polymorphism and essential hypertension in a Japanese population. Am J Hypertens. 1998;11:5026.

43. Larson N, Hutchinson R, Boerwinkle E. Lack of association of 3 functional gene variants with hypertension in African Americans. Hypertension. 2000;35:1297-300.

44. He X, Zhu DL, Chu SL, Jin L, Xiong MM, Wang GL, et al. Alfa-Adducin gene and essential hypertension in China. Clin Exp Hypertens. 2001;23:579-89.

45. Kaiser HW, O'Keefe E, Bennett V. Adducin: Ca++ - dependent association with sites of cell-cell contact. J Cell Biol. 1989;109:557-69.

46. Tripodi G, Valtorta F, Torielli L, Chieregatti E, Salardi S, Trusolino L, et al. Hypertension-associated point mutations in the adducin alpha and beta subunits affect actin cytoskeleton and ion transport. J. Clin Invest. 1996;97:2815-22.

47. Manunta P, Burnier M, D’Amico M, Buzzi L, Maillard M, Barlassina C, et al. Adducin polymorphism affects renal proximal tubule reabsorption in hypertension. Hypertension. 1999;33:694-7.

48. Siffert W, Rosskopf D, Siffert G, Busch S, Moritz A, Erbel R, et al. Association of a human G-protein beta3 subunit variant with hypertension. Nat Genet. 1998;18:45-8. 
49. Benjafield AV, Jeyasingam CL, Nyholt DR, Griffiths LR, Morris BJ. G-Protein beta3 subunit Gene (GNB3) variant in causation of essential hypertension. Hypertension. 1998;32:1094-7.

50. Li M, Zhang B, Li C, Liu JL, Wang LJ, Liu Y, et al. G-protein beta 3 subunit polymorphisms and essential hypertension: a case-control association study in northern Han Chinese. J Geriatr Cardiol. 2015;12:127-34.

51. Zheng $\mathrm{H}$, Xu H, Cui B, Xie N, Wang Z, Luo M. Association between polymorphism of the G-protein $\beta 3$ subunit C825T and essential hypertension: an updated meta-analysis involving 36,802 subjects. Biol Res. 2013;46:265-73.

52. Brand E, Herrmann SM, Nicaud V, Ruidavets JB, Evans A, Arveiler D, et al. The $825 \mathrm{C} / \mathrm{T}$ polymorphism of the G-protein subunit, beta 3 is not related to hypertension. Hypertension. 1999;33:1175-8.

53. Kato N, Sugiyama T, Morita H, Kurihara H, Yamori Y, Yazaki Y. G protein beta3 subunit variant and essential hypertension in Japanese. Hypertension. 1998;32:935-8.

54. Levine MA, Modi WS, O'Brien SJ. Chromosomal localization of the genes encoding two forms of the $\mathrm{G}$ protein beta polypeptide, beta 1 and beta 3, in man. Genomics. 1990;8:380-6.

55. Siffert W. G proteins, hypertension, and coronary heart disease - novel findings and hypotheses. Kidney Blood Press Res. 1996;19:71-80.

56. Siffert W, Düsing R. Sodium-proton exchange and primary hypertension: an update. Hypertension. 1995;26:649-55.

57. Gonçalves R, Freitas A, Branco M, Rosa A, Fernandes AT, Zhivotovsky LA, et al. Y-chromosome lineages from Portugal, Madeira and Açores record elements of Sephardim and Berber ancestry. Ann Hum Genet. 2005;69:443-54.

58. Brehm A, Pereira L, Kivisild T, Amorim A. Mitochondrial portraits of the Madeira and Açores archipelagos witness different genetic pools of its settlers. Hum Genet. 2003;114:77-86.

59. Kristiansson K, Naukkarinen J, Peltonen L. Isolated populations and complex disease gene identification. Genome Biol. 2008;9:109.

60. Jorde LB, Wooding SP. Genetic variation, classification and race. Nat Genet. 2004;36:S28-33. 